\title{
Excitons at a single localized center induced by a natural composition modulation in bulk $\mathrm{Ga}_{0.5} \mathbf{I n}_{0.5} \mathrm{P}$
}

\author{
Mitsuru Sugisaki* and Hong-Wen Ren ${ }^{\dagger}$ \\ Single Quantum Dot Project, ERATO, JST, Tsukuba Research Consortium, 5-9-9 Tokodai, Tsukuba, Ibaraki 300-2635, Japan \\ Kenichi Nishi and Shigeo Sugou \\ Single Quantum Dot Project, ERATO, JST, Tsukuba Research Consortium, 5-9-9 Tokodai, Tsukuba, Ibaraki 300-2635, Japan \\ and Optoelectronics and High Frequency Device Research Laboratories, NEC Corporation, 34 Miyukigaoka, Tsukuba, \\ Ibaraki 305-8501, Japan \\ Yasuaki Masumoto \\ Single Quantum Dot Project, ERATO, JST, Tsukuba Research Consortium, 5-9-9 Tokodai, Tsukuba, Ibaraki 300-2635, Japan \\ and Institute of Physics, University of Tsukuba, Tsukuba, Ibaraki 305-8571, Japan
}

(Received 27 December 1999)

\begin{abstract}
Optical properties of $\mathrm{Ga}_{0.5} \mathrm{In}_{0.5} \mathrm{P}$ grown by gas-source molecular beam epitaxy are reported. A strong optical anisotropy due to the spontaneous lateral composition modulation of $\mathrm{Ga}$ and In was observed in macro- and micro-photoluminescence (PL) spectra. The micro-PL study revealed that the PL band is composed of sharp lines that arise from the radiative decay of excitons trapped at local potential minima caused by the composition modulation. Anisotropy in PL rise and decay times was also observed. The detection energy dependence of the PL decay time is well explained by considering the relaxation process of excitons from shallow traps to deep traps.
\end{abstract}

\section{INTRODUCTION}

With the development of well-controlled fabrication techniques, ternary alloy semiconductors of III-V compound have been extensively studied because of the fundamental interest and its technological potential. In particular, the direct gap $\mathrm{Ga}_{x} \operatorname{In}_{1-x} \mathrm{P}$ semiconductor is widely used for light emitting devices in the red wavelength region. It is well known that $\mathrm{Ga}_{x} \operatorname{In}_{1-x} \mathrm{P}$ grown by a metal-organic vapor phase epitaxy (MOVPE) system exhibits natural superlattice ordering characterized by alternating Ga-rich and In-rich monolayers. By using this property, novel optodevices, such as a low threshold laser and polarization switch, have been proposed. ${ }^{1-3}$ From the basic viewpoint, the band-gap reduction and optical anisotropy induced by the composition modulation of $\mathrm{Ga}$ and In are interesting subjects.

$\mathrm{Ga}_{0.5} \mathrm{In}_{0.5} \mathrm{P}$ lattice-matched to a GaAs substrate is generally used as the matrix for InP self-assembled quantum dots as well. ${ }^{4}$ Lately it was reported that $\mathrm{Ga}_{0.5} \mathrm{In}_{0.5} \mathrm{P}$ grown by a gas-source molecular beam epitaxy (GS MBE) exhibits lateral composition modulation, and that it causes a strong optical anisotropy in InP self-assembled quantum dots. ${ }^{5-7}$ In contrast to many reports of the natural superlattice formation in bulk $\mathrm{Ga}_{0.5} \mathrm{In}_{0.5} \mathrm{P}$, the study on the natural composition modulation has just begun. ${ }^{8}$ It is well known that lateral composition modulation spontaneously occurs when a short period superlattice is fabricated. ${ }^{9-12}$ Some authors have reported that the natural composition modulation arises even in bulk ternary and quaternary alloy semiconductors, such as $\mathrm{InAs}_{x} \mathrm{Sb}_{1-x}$ (Ref. 13), $\mathrm{In}_{1-x} \mathrm{Ga}_{x} \mathrm{As}_{y} \mathrm{P}_{1-y}$ (Ref. 14), and $\mathrm{In}_{x} \mathrm{Al}_{1-x} \mathrm{As}$ (Ref. 15). In order to understand the electronic structure of such systems, it is important to investigate the optical properties from the microscopic point of view because the crystal field potential fluctuates on the same scale as the composition modulation. This will also help to achieve a better understanding of carrier dynamics in self-assembled quantum dots or quantum wires embedded in a matrix with lateral composition modulation.

In this paper, we report the optical properties of $\mathrm{Ga}_{0.5} \mathrm{In}_{0.5} \mathrm{P}$ in submicrometer region, and discuss the relaxation process of excitons trapped at single localized centers in the presence of potential fluctuation.

\section{EXPERIMENTAL DETAILS}

Growth of $\mathrm{Ga}_{0.5} \mathrm{In}_{0.5} \mathrm{P}$ was carried out on GaAs (001) substrates using a GS MBE system. ${ }^{5}$ After the growth of a GaAs buffer layer, a 500-nm-thick $\mathrm{Ga}_{0.5} \mathrm{In}_{0.5} \mathrm{P}$ layer was formed at $500{ }^{\circ} \mathrm{C}$. Conventional macro-photoluminescence (PL) and time-resolved PL measurements were performed using second harmonic pulses of a mode-locked Ti:sapphire laser with pulse duration $\sim 5 \mathrm{ps}$ and repetition rate $82 \mathrm{MHz}$. The specimen was immersed directly into superfluid He. The sample PL was fed to a 50-cm single monochromator using an optical fiber and detected by a liquid nitrogen cooled charge coupled device (CCD) camera. The spectral resolution of this system is better than $0.3 \mathrm{meV}$. The measurement system of the PL decay time is composed of a $25-\mathrm{cm}$ subtractive dispersion double monochromator and a synchroscan streak camera. The time resolution of the system is about 30 ps.

In order to measure the micro-PL ( $\mu$-PL) spectra, micro patterns were drawn on the sample by means of photolithography. ${ }^{16}$ After wet etching by $\mathrm{HCl}: \mathrm{H}_{2} \mathrm{O}=2: 1$ at $30{ }^{\circ} \mathrm{C}$, the mesa size was reduced to about $\phi 1 \mu \mathrm{m}$. The 


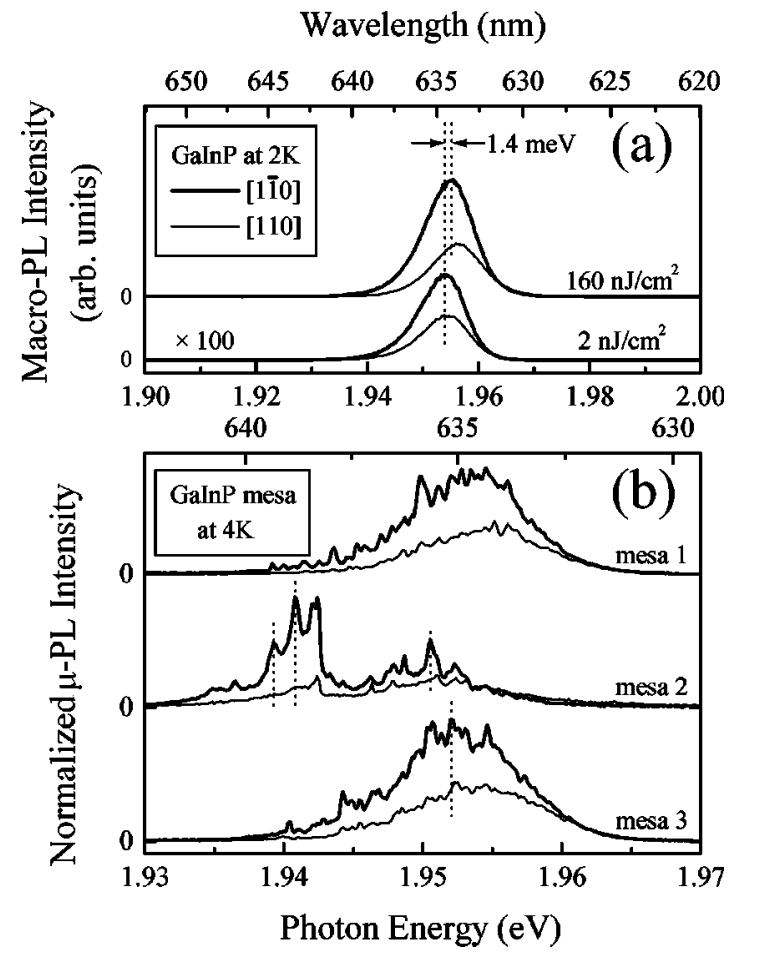

FIG. 1. (a) Excitation power dependence of macro-PL spectra at $2 \mathrm{~K}$ observed for the [110] and [1̄o] polarizations. (b) $\mu$-PL spectra near the macro-PL peak in three different mesas. The area of the mesa is $\phi \sim 1 \mu \mathrm{m}$. The sharp PL lines observed around 1.95 $\mathrm{eV}$ are considered to arise from excitons trapped at local minima formed due to spontaneous lateral composition modulation.

sample was set on a cold-finger of a liquid He flow-type cryostat, and then excited by a continuous wave (cw) Ar-ion laser. The PL from the sample was collected using a microscope objective lens with a numerical aperture of 0.42 , and analyzed by a $50-\mathrm{cm}$ single monochromator and a CCD camera.

\section{RESULTS AND DISCUSSION}

The thick and thin curves in Fig. 1(a) show the time integrated macro-PL spectra observed at $2 \mathrm{~K}$ polarized along the [11 0$]$ and [110] directions of the GaAs substrate, respectively. The PL peak from $\mathrm{Ga}_{0.5} \mathrm{In}_{0.5} \mathrm{P}$ measured under weak excitation $\left(2 \mathrm{~nJ} / \mathrm{cm}^{2}\right)$ was observed at $1.954 \mathrm{eV}$ with a bandwidth of $9.8 \mathrm{meV}$, which is the same as that observed under weak cw Ar-ion laser excitation. The degree of linear polarization $P$ defined by

$$
P=\frac{I_{[1 \overline{1} 0]}-I_{[110]}}{I_{[1 \overline{1} 0]}+I_{[110]}}
$$

is $32 \%$ at the PL peak, where $I_{[1 \overline{1} 0]}$ and $I_{[110]}$ are the PL intensities observed for the [1 10$]$ and [110] polarizations, respectively.

The band-gap energy of the completely disordered $\mathrm{Ga}_{0.5} \mathrm{In}_{0.5} \mathrm{P}$ is $2.005 \mathrm{eV}$ at $4 \mathrm{~K},{ }^{17}$ but the observed PL peak energy is lower by about $50 \mathrm{meV}$ than this value. Crosssectional transmission electron microscopy (XTEM) revealed that the optical anisotropy is due to alternative lateral composition modulation of $\mathrm{Ga}$ and In along the [110] (a)

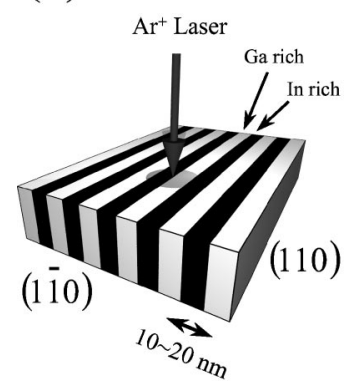

(b)

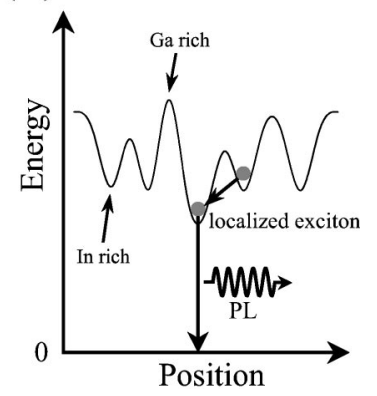

FIG. 2. (a) Schematic drawing of the $\mathrm{Ga}_{0.5} \mathrm{In}_{0.5} \mathrm{P}$ sample forming the spontaneous lateral composition modulation. In-rich and Garich domains align alternately along the $[110]$ direction. The modulation planes are parallel to [1 $1 \overline{0} 0]$ direction with the separation of about 10-20 nm. For the optical measurements, the (001) surface was used. (b) Schematic representation of the band structure. In the real system, the thickness of modulation planes and the modulation amplitude slightly differ from site to site, resulting in the fluctuation of the localized energies of the excitons. The excitons are considered to relax successively by phonon emission.

direction, ${ }^{5}$ as schematically shown in Fig. 2(a). The modulation period is about $10-20 \mathrm{~nm}$. The PL intensity is strong when the observed polarization is along the modulation planes, i.e., the $[1 \overline{1} 0]$ direction. We prepared several samples grown at various temperatures, and found that the sample having smaller band gap, which indicates the sample has large composition modulation, shows stronger optical anisotropy. ${ }^{5}$

As shown in Fig. 1(a), when the excitation power is increased by about 100 times, the PL peak energies show a small but clear blueshift observed for both polarization constituents, $1.4 \mathrm{meV}$ for the [1 10$]$ polarization and $2.2 \mathrm{meV}$ for the [110] polarization. These results suggest that the PL peak from $\mathrm{Ga}_{0.5} \mathrm{In}_{0.5} \mathrm{P}$ arises from excitons trapped at local minima. In a low-dimensional system such as quantum dots, as the excitation power is increased, lower-energy states of the confined exciton get occupied and the PL from the higher-energy exciton states becomes dominant. This is called state filling effect. A high-energy shift under high power excitation has been observed in some ternary alloy systems. ${ }^{18,19}$ The degree of linear polarization under high power excitation is $36 \%$, which is slightly higher than that observed under weak excitation. This result suggests that the higher-energy exciton states have a stronger optical anisotropy.

The local potential minima in the $\mathrm{Ga}_{0.5} \mathrm{In}_{0.5} \mathrm{P}$ is possibly caused by the lateral composition modulation with a lateral extension of a few tens of nanometers as mentioned above. In the real system, the thickness of modulation planes and the modulation amplitude slightly differ from site to site, resulting in the fluctuation of the local potential minimum, as schematically shown in Fig. 2(b).

In order to confirm directly the existence of local potential minima, a $\mu$-PL measurement was performed. Figure 1(b) shows the polarization dependence of the $\mu$-PL spectra observed in three different mesas. The experiments were performed under weak excitation in order to eliminate the state filling effect since it causes the line broadening due to 


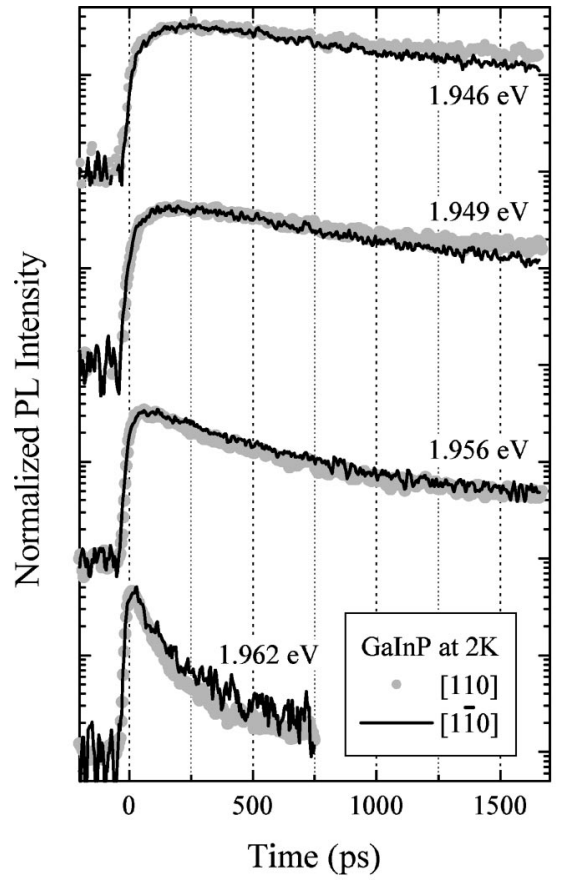

FIG. 3. Polarized time-resolved PL curves observed at 1.946 $\mathrm{eV}, 1.949 \mathrm{eV}, 1.956 \mathrm{eV}$, and $1.962 \mathrm{eV}$. Data plotted by the circles and solid lines were observed for the [110] and [1 $\overline{1} 0]$ polarizations, respectively.

carrier-carrier interaction, and thus makes the result ambiguous. Very sharp PL lines, which resemble to excitons confined in single quantum dots, were observed with peak energies differing from mesa to mesa. We consider that these distinct lines come from the radiative decay of excitons trapped at the local potential minimum formed by the lateral composition modulation. ${ }^{20}$ The PL peak from the exciton trapped at a shallow trap appears at the high-energy side, while that from deep center is observed at the low-energy side.

As in the case of the macro-PL spectra, the $\mu$-PL intensity is always stronger when observed for the [1 10$]$ polarization. Further, as marked by dotted lines, some PL lines seem to have different energy between the [1 $1 \overline{1} 0]$ and [110] polarizations. This result indicates that each localized center has an anisotropic electronic structure, and an ensemble of the sharp line gives the broad PL band and the optical anisotropy in the macro-PL spectra as shown in Fig. 1(a).

From a comparison with a theoretical report by Mattila et al. ${ }^{21}$ it is considered that the sharp PL lines come from the radiative recombination of electrons and holes at an In-rich site [see Fig. 2(b)]. The polarization dependence observed in this study agrees with their calculation, i.e., the PL intensity is strong when observed for the [1 $1 \overline{0} 0]$ polarization. They also mentioned that this system has a type I character, i.e., direct in coordinate and momentum spaces, which is consistent with fast PL decay times reported below (Figs. 3 and 4).

Figure 3 shows temporal change of the PL intensity of the $\mathrm{Ga}_{0.5} \mathrm{In}_{0.5} \mathrm{P}$ band observed for the $[1 \overline{1} 0]$ and [110] polarizations. Each PL decay curve is normalized at the peak. The PL decay time of the $\mathrm{Ga}_{0.5} \mathrm{In}_{0.5} \mathrm{P}$ with the lateral composition modulation is briefly reported in Ref. 22. The excitation power was reduced as much as possible [the same as the

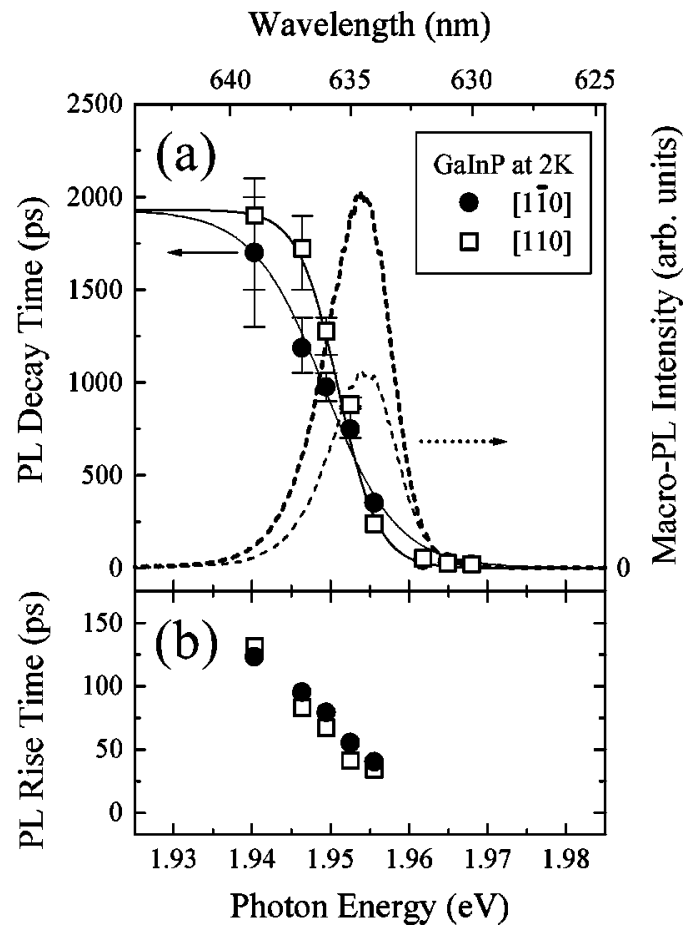

FIG. 4. PL (a) decay time and (b) rise time obtained using Eq. (2). The thick and thin dotted curves are the macro-PL spectra observed for the [1 $1 \overline{1} 0]$ and [110] polarizations, respectively. As shown by the solid lines, the detection energy dependence of the PL decay times can be well fitted using Eq. (3).

lower spectra of Fig. 1(a)]. Apparently, the PL decay time is much faster than that in $\mathrm{Ga}_{0.5} \mathrm{In}_{0.5} \mathrm{P}$ containing the $\mathrm{Cu}-\mathrm{Pt}_{\mathrm{B}}$ type natural superlattice. ${ }^{23,24} \mathrm{~A}$ slight but certain polarization dependence of the PL decay time is observed. The PL decay time observed for the [110] polarization is faster when observed above the PL peak energy of the $\mathrm{Ga}_{0.5} \mathrm{In}_{0.5} \mathrm{P}$ band, while that observed for the [1 $1 \overline{0} 0]$ polarization is faster when observed at the low-energy side of the PL band.

By assuming a double exponential decay profile, the PL decay time $\tau_{\text {fd }}$ and $\tau_{\text {sd }}$ and the rise time $\tau_{\mathrm{r}}$ are estimated by fitting the observed curve as

$$
I(t)=\left[1-\exp \left(-t / \tau_{\mathrm{r}}\right)\right]\left[A_{\mathrm{f}} \exp \left(-t / \tau_{\mathrm{fd}}\right)+A_{\mathrm{s}} \exp \left(-t / \tau_{\mathrm{sd}}\right)\right],
$$

where, $A_{\mathrm{f}}$ and $A_{\mathrm{s}}$ are constants. Unfortunately, the origin of the slow decay component $\left[\exp \left(-t / \tau_{\mathrm{sd}}\right)\right]$ is not clarified yet. Hereafter we discuss the fast decay component [exp $\left.\left(-t / \tau_{\mathrm{fd}}\right)\right]$ of the PL decay profile.

As shown in Fig. 4(a), the PL decay time observed for each polarization becomes gradually longer as the detection energy is decreased. This is the typical behavior of the PL decay time in localized excitons. ${ }^{19,25-27}$ On the other hand, the PL rise time observed at $1.962 \mathrm{eV}$ was as fast as the laser pulse width, but it became slower as the detection energy was decreased as shown in Fig. 4(b). From these results, it is concluded that the excitons trapped by a shallow local minimum successively relax to deeper trap centers, probably by phonon emission [see Fig. 2(b)].

This assumption is supported by the exciton transfer model. ${ }^{27-29}$ As shown by solid lines in Fig. 4(a), the PL decay times $\tau_{\mathrm{fd}}$ observed for the [1 $\left.1 \overline{1} 0\right]$ and [110] polariza- 
tions are well fitted using this model, which gives $\tau_{\mathrm{fd}}$ as a function of emission energy $E$ as

$$
\tau_{\mathrm{fd}}(E)=\frac{\tau_{\mathrm{rd}}}{1+\exp \left[\left(E-E_{\mathrm{em}}\right) / E_{0}\right]}
$$

where $\tau_{\text {rd }}$ represents the radiative lifetime and $E_{\mathrm{em}}$ stands for the characteristic energy that is analogous to the mobility edge. For simplicity, the density of states is assumed to be proportional to $\exp \left(-E / E_{0}\right)$, i.e., $E_{0}$ is a measure of the depth in the localized state. Rather good fittings were obtained using the following parameters: $\tau_{\mathrm{rd}}=1930 \pm 80 \mathrm{ps}, E_{\mathrm{em}}$ $=1.9515 \mathrm{eV} \pm 0.5 \mathrm{meV}$, and $E_{0}=2.54 \pm 0.4 \mathrm{meV}$ for the [110] polarization, and $\tau_{\mathrm{rd}}=1930 \pm 50 \mathrm{ps}, E_{\mathrm{em}}$ $=1.9493 \mathrm{eV} \pm 0.4 \mathrm{meV}$, and $E_{0}=4.59 \pm 0.4 \mathrm{meV}$ for the [1 10$]$ polarization.

The PL intensity is considered to be proportional to the density of states. Since the estimated $E_{0}$ for the [1 10$]$ polarization is about twice larger than that for the [110] polarization, the density of states for the [1 10$]$ polarization also becomes higher. Therefore efficient radiative decay of excitons is excepted when observed for the [1 $1 \overline{1} 0]$ polarization. This is qualitatively in good agreement with the results shown in Figs. 1(a) and 1(b).

\section{SUMMARY}

We studied the optical properties of $\mathrm{Ga}_{0.5} \mathrm{In}_{0.5} \mathrm{P}$ grown by means of a GS MBE. A strong optical anisotropy was observed in macro- and $\mu$-PL spectra. From XTEM study, the optical anisotropy is found to be due to the spontaneous lateral composition modulation of Ga and In. The $\mu$-PL study revealed that the PL band is composed of many of sharp lines, which come from the radiative decay of excitons trapped at local potential minima. Anisotropy in the PL rise and decay times was also observed. The detection energy dependence of the PL decay time is well explained by considering the relaxation process of excitons from shallow traps to deep traps. All these observed results indicate that, for the study on the relaxation process of excitons in this system, it is important to consider the localized excitons due to the anisotropic potential minima induced by the spontaneous lateral composition modulation.

Note added in proof. Recently, some reports on the observation of the $\mu$-PL lines in bulk alloy semiconductors were published. ${ }^{30,31}$

\section{ACKNOWLEDGMENTS}

The authors wish to thank Dr. S. V. Nair and T. Okuno for valuable discussions and a critical reading of the manuscript.
*Electronic address: mitsuru@sqdp.trc-net.co.jp

†Present address: Space Vacuum Epitaxy Center, University of Houston, 4800 Calhoun, Houston, TX 77204-5507.

${ }^{1}$ T. Kippenberg, J. Krauss, J. Spieler, P. Kiesel, E. Greger, M. Moser, and G.H. Döhler, IEEE Photonics Technol. Lett. 11, 427 (1999); E. Greger, P. Reil, M. Moser, T. Kippenberg, P. Kiesel, and G. H. Döhler, Appl. Phys. Lett. 71, 3245 (1997).

${ }^{2}$ P.D. Floyd, D. Sun, and D.W. Treat, IEEE Photonics Technol. Lett. 10, 45 (1998).

${ }^{3}$ F. Scholz, C. Geng, M. Burkard, H.-P. Gauggel, H. Schweizer, R. Wirth, A. Moritz, and A. Hangleiter, Physica E (Amsterdam) 2, 8 (1998).

${ }^{4}$ For a review article, see A. Gustafsson, M.-E. Pistol, L. Montelius, and L. Samuelson, J. Appl. Phys. 84, 1715 (1998), and references therein. See also P.M. Petroff and S.P. DenBaars, Superlattices Microstruct. 15, 15 (1994).

${ }^{5}$ H.-W. Ren, M. Sugisaki, S. Sugou, K. Nishi, A. Gomyo, and Y. Masumoto, Jpn. J. Appl. Phys., Part 1 38, 2438 (1999).

${ }^{6}$ M. Sugisaki, H.-W. Ren, S.V. Nair, K. Nishi, S. Sugou, T. Okuno, and Y. Masumoto, Phys. Rev. B 59, R5300 (1999); M. Sugisaki, H.-W. Ren, S.V. Nair, K. Nishi, S. Sugou, T. Okuno, and Y. Masumoto, in Excitonic Processes in Condensed Matter, edited by R. T. Williams and W.M. Yen (The Electrochemical Society Proceedings Series, Pennington, NJ, 1998), Vol. 98-25, p. 298.

${ }^{7}$ M. Sugisaki, H.-W. Ren, E. Tokunaga, K. Nishi, S. Sugou, T. Okuno, and Y. Masumoto, in Proceedings of the 24th International Conference on the Physics of Semiconductors, edited by D. Gershoni (World Scientific, Singapore, 1999), PDF No. 1180.

${ }^{8}$ As a review article, Optoelectronic Materials: Ordering, Composition Modulation, and Self-Assembled Structures, edited by D. Jones, A. Mascarenhas, and P. Petroff (Material Research Society, Pittsburgh, Pennsylvania, 1996).
${ }^{9}$ P.J. Pearah, A.C. Chen, A.M. Moy, K.C. Hsieh, and K.Y. Cheng, J. Cryst. Growth 127, 900 (1993).

${ }^{10}$ S.T. Chuo, K.Y. Cheng, L.J. Chuo, and K.C. Hsieh, J. Appl. Phys. 78, 6270 (1995).

${ }^{11}$ Y. Tang, H.T. Lin, D.H. Rich, P. Colter, and S.M. Vernon, Phys. Rev. B 53, R10 501 (1996).

${ }^{12}$ J.M. Millunchick, R.D. Twesten, D.M. Follstaedt, S.R. Lee, E.D. Jones, Y. Zhang, S.P. Ahrenkiel, and A. Mascarenhas, Appl. Phys. Lett. 70, 1402 (1997).

${ }^{13}$ T.-Y. Seong, A.G. Norman, I.T. Ferguson, and G.R. Booker, J. Appl. Phys. 73, 8227 (1993).

${ }^{14}$ R.R. LaPierre, T. Okada, B.J. Robinson, D.A. Thompson, and G.C. Weatherly, J. Cryst. Growth 158, 6 (1996).

${ }^{15}$ S.W. Jun, T.-Y. Seong, J.H. Lee, and B. Lee, Appl. Phys. Lett. 68, 3443 (1996).

${ }^{16}$ M. Sugisaki, H.-W. Ren, S. Sugou, K. Nishi, and Y. Masumoto, Solid-State Electron. 42, 1325 (1998); M. Sugisaki, H.-W. Ren, K. Nishi, S. Sugou, T. Okuno, and Y. Masumoto, Physica B 256-258, 169 (1998).

${ }^{17}$ M.C. DeLong, D.J. Mowbray, R.A. Hogg, M.S. Skolnick, J.E. Williams, K. Meehan, S.R. Kurtx, J.M. Olson, R.P. Schneider, M.C. Wu, and M. Hopkinson, Appl. Phys. Lett. 66, 3185 (1995).

${ }^{18}$ M.C. DeLong, P.C. Taylor, and J.M. Olson, Appl. Phys. Lett. 57, 620 (1990).

${ }^{19}$ Y. Narukawa, Y. Kawakami, Sz. Fujita, Sg. Fujita, and S. Nakamura, Phys. Rev. B 55, R1938 (1997).

${ }^{20}$ Sharp $\mu$-PL lines from localized excitons are also observed in $\mathrm{Ga}_{0.5} \mathrm{In}_{0.5} \mathrm{P}$ containing the $\mathrm{Cu}-\mathrm{Pt}_{\mathrm{B}}$ type long-range ordering domains grown using MOVPE system. Details will be reported elsewhere.

${ }^{21}$ T. Mattila, L.-W. Wang, and A. Zunger, Phys. Rev. B 59, 15270 (1999).

${ }^{22}$ T. Okuno, H.-W. Ren, M. Sugisaki, K. Nishi, S. Sugou, and Y. 
Masumoto, Phys. Rev. B 57, 1386 (1998).

${ }^{23}$ M.C. DeLong, W.D. Ohlsen, I. Viohl, P.C. Taylor, and J.M. Olson, J. Appl. Phys. 70, 2780 (1991).

${ }^{24}$ T. Kobayashi, M. Minaki, K. Takashima, K. Uchida, and J. Nakahara, Phys. Status Solidi B 198, 49 (1996).

${ }^{25}$ Y. Kawakami, M. Funato, Sz. Fujita, Sg. Fujita, Y. Yamada, and Y. Masumoto, Phys. Rev. B 50, 14655 (1994).

${ }^{26}$ S. Yamaguchi, Y. Kawakami, Sz. Fujita, Sg. Fujita, Y. Yamada, T. Mishina, and Y. Masumoto, Phys. Rev. B 54, 2629 (1996).

${ }^{27}$ C. Gourdon and P. Lavallard, Phys. Status Solidi B 153, 641
(1989); C. Gourdon and P. Lavallard, J. Cryst. Growth 101, 767 (1990).

${ }^{28}$ E. Cohen and M.D. Sturge, Phys. Rev. B 25, 3828 (1982).

${ }^{29}$ M. Oueslati, C. Benoit á la Guillaume, and M. Zouaghi, Phys. Rev. B 37, 3037 (1988).

${ }^{30}$ U. Kops, P. G. Blome, M. Wenderoth, R. G. Ulbrich, C. Geng, and R. Scholz, Phys. Rev. B 61, 1992 (2000).

${ }^{31}$ U. Dörr, R. Lutz, J. Schuler, H. Kalt, W. Send, and D. Gerthsen, J. Lumin. 87-89, 718 (2000). 